\title{
Three novel insect-associated species of Simplicillium (Cordycipitaceae, Hypocreales) from Southwest China
}

\author{
Wan-Hao Chen', Chang Liu², Yan-Feng Han³, \\ Jian-Dong Liang', Wei-Yi Tian', Zong-Qi Liang ${ }^{3}$
}

I Department of Microbiology, Basic Medical School, Guizhou University of Traditional Chinese Medicine, Guiyang 550025, Guizhou, China 2 School of Pharmacy, Guizhou University of Traditional Chinese Medicine, Guiyang 550025, Guizhou, China 3 Institute of Fungus Resources, Department of Ecology, College of Life Sciences, Guizhou University, Guiyang 550025, Guizhou, China

Corresponding author: Yan-Feng Han (swallow1128@126.com)

Academic editor:Nalin Wijayawardene $\mid$ Received 12 June 2019|Accepted 24August 2019| Published25 September 2019

Citation: Chen W-H, Liu C, Han Y-F, Liang J-D, Tian W-Y, Liang Z-Q (2019) Three novel insect-associated species of Simplicillium (Cordycipitaceae, Hypocreales) from Southwest China. MycoKeys 58: 83-102. https://doi. org/10.3897/mycokeys.58.37176

\begin{abstract}
In this paper, we introduce three new species of Simplicillium, viz. S. cicadellidae, S. formicidae and S. lepidopterorum, which were isolated from an infected leafhopper, ant and carpenterworm, respectively. Morphological comparisons and phylogenetic analyses based on multigene datasets (LSU+RPB1+RPB2+TEF and ITS+LSU) support the establishment of the three new species. Simplicillium cicadellidae was distinguished from other species in morphological characteristics by having smaller phialides and ellipsoidal conidia, and lacking octahedral crystals. The reverse of colonies were yellowish (\#FFBF00), especially in the middle, and radially sulcate. Simplicillium formicidae was morphologically distinguished from other by having longer phialides and filiform to fusoid conidia, and by lacking octahedral crystals. Simplicillium lepidopterorum was morphologically distinguished from other species by having smaller, ellipsoidal to fusiform conidia, and by lacking octahedral crystals. The reverse of the colony was pale white. The three new species are likely to be nourished by plant to animal (especially insect) nutrients based on the evolutionary pattern of the Hypocreales, and they are described herein as being clearly distinct from other species in Simplicillium.
\end{abstract}

\section{Keywords}

Commensal fungi, morphology, nutritional preference, phylogeny

Copyright Wan-Hao Chen et al. This is an open access article distributed under the terms of the Creative Commons Attribution License (CC BY 4.0), which permits unrestricted use, distribution, and reproduction in any medium, provided the original author and source are credited. 


\section{Introduction}

The genus Simplicillium W. Gams \& Zare was introduced by Zare and Gams (2001) with S. lanosoniveum (J. F. H. Beyma) Zare \& W. Gams as the type species. The genus is characterized with its complete lack of verticillate branching; mostly solitary phialides, which are discrete, aculeate and narrow and arise from aerial hyphae; conidia short-ellipsoidal to suglobose or obclavate, and adhering in globose heads or imbricate chains (Zare and Gams 2001). The members of Simplicillium are fungicolous and occur on various substrata (Zare and Gams 2001; Chen et al. 2008; Baiswar et al. 2014; Gauthier et al. 2014; Gomes et al. 2018). Furthermore, Zare and Gams (2001) introduced three additional species, viz., S. lamellicola (F. E. V. Sm.) Zare \& W. Gams, S. obclavatum (W. Gams) Zare \& W. Gams and S. wallacei H. C. Evans. The typical characteristics of Simplicillium include mostly solitary phialides, conidia adhering in globose, slimy heads or imbricate chains, and commonly present crystals in the agar (Zare and Gams 2001). Later, Zare and Gams (2008) transferred S. wallacei to Lecanicillium W. Gams \& Zare based on the phylogenic analysis of internal transcribed spacer (ITS) region and this transfer was confirmed by Sung et al. (2007).

Liu and Cai (2012) reported a new species, S. chinense F. Liu \& L. Cai, which was the first Simplicillium species from China. Five new Simplicillium species, S. aogashimaense Nonaka, Kaifuchi \& Masuma, S. cylindrosporum Nonaka, Kaifuchi \& Masuma, S. minatense Nonaka, Kaifuchi \& Masuma, S. subtropicum Nonaka, Kaifuchi \& Masuma and S. sympodiophorum Nonaka, Kaifuchi \& Masuma were reported by Nonaka et al. (2013) from Tokyo, Japan. Simplicillium calcicola Z. F. Zhang, F. Liu \& L. Cai, S. coffeanum A. A. M. Gomes \& O. L. Pereira and S. filiforme R. M. F. Silva, R. J. V. Oliveira, Souza-Motta, J. L. Bezerra \& G. A. Silva were reported by Zhang et al. (2017), Gomes et al. (2018) and Crous et al. (2018), respectively. Currently, Simplicillium consists of 12 species.

Kepler et al. (2017) re-evaluated the Cordycipitaceae based on the multigene dataset (SSU, LSU, TEF, RPB1 and RPB2), and indicated that Simplicillium species group in a clade and are the earliest diverging lineage in Cordycipitaceae. The nuclear ribosomal ITS and LSU were first used to identify cryptic diversification among Simplicillium species by Liu and Cai (2012) and then were widely applied in the identification of Simplicillium species by Nonaka et al. (2013), Zhang et al. (2017), Gomes et al. (2018) and Crous et al. (2018).

Zare and Gams (2001) noted that Simplicillium species were found on various substrata and fungi. Other substrata were found later, such as limstone and wood (Liu and Cai 2012; Zhang et al. 2017). Many bioactive compounds were discovered in Simplicillium, such as alkaloids (Fukuda et al. 2014), peptides (Liang et al. 2016; 2017; Dai et al. 2018), diketopiperazine (Yan et al. 2015), xylanases (Roy et al. 2013), anthraquinones (Huang et al. 2015), antibiotics (Takata et al. 2013; Dong et al. 2018), and especially Simpotentin, which is a new potentiator of amphotericin B activity against Candida albicans (C. P. Robin) Berkhout and has showed great potential ap- 
plications in medicine (Uchida et al. 2019). Furthermore, the antimicrobial activities and entomopathogenicity has meant that Simplicillium has potential applications in biocontrol (Ward et al. 2012; Zhao et al. 2013; Le Dang et al. 2014; Lim et al. 2014; Chen et al. 2017; Skaptsov et al. 2017). However, as far as we know, there are limited reports of Simplicillium species isolated from infected insects.

Three infected insect specimens were found during a survey of araneogenous fungi and allies from southwestern China. Some fungal strains were isolated and purified from the three specimens. Based on polyphasic approach (morphological, ecological characteristics along with a phylogenetic analysis), they were identified as three new species, Simplicillium cicadellidae sp. nov., S. formicidae sp. nov. and S. lepidopterorum sp. nov.

\section{Materials and methods}

\section{Collection and isolation}

Three infected insect specimens (DL1004, GY1101 and GY2913) were collected from Dali, Rongjiang Country $\left(26^{\circ} 01^{\prime} 58.70^{\prime \prime N}, 108^{\circ} 24^{\prime} 48.06^{\prime \prime E}\right)$ and Tongmuling (26'ㄹ' 25.92"N, 106 $\left.41^{\prime} 3.35^{\prime \prime E}\right)$, Huaxi District, Guizhou Province, on 1 October, 9 November and 31 July, 2018, respectively. The surface of the specimens were rinsed with sterile water, followed by surface sterilization with $75 \%$ ethanol for 3-5 s. A part of the insect body was cut off and used to inoculate a piece of tissue in haemocoel on potato dextrose agar (PDA) and improved potato dextrose agar (PDA, $1 \% \mathrm{w} / \mathrm{v}$ peptone) ( $\mathrm{Qu}$ et al. 2018). The strain was isolated and cultured at $22^{\circ} \mathrm{C}$ for $14 \mathrm{~d}$ under $12 \mathrm{~h}$ light/12 h dark conditions following protocols described by Zou et al. (2010). Strains DL10041, DL10042, GY11011, GY11012, GY29131 and GY29132 were obtained.

\section{Culture and identification}

The strains were incubated in PDA at $25^{\circ} \mathrm{C}$ for $14 \mathrm{~d}$. Macroscopic and microscopic morphological characteristics of the fungi were examined using classical mycological techniques, and the growth rates were determined. The fresh hyphae were observed with an optical microscope (OM, BX35, Olympus, Japan) following pretreatment with lactophenol cotton blue solution or normal saline. The ex-type cultures and dried culture as holotype specimens were deposited in GZAC, Guizhou University, Guiyang, China.

\section{DNA extraction, PCR amplification and nucleotide sequencing}

DNA extraction was carried out in accordance with Liang et al. (2009). The extracted DNA was stored at $-20{ }^{\circ} \mathrm{C}$. The amplification of large subunit ribosomal 
RNA (LSU) genes was performed using NS1-1/AB28 primers (Curran et al. 1994). Translation elongation factor 1 alpha (TEF) and DNA-directed RNA polymerase II largest subunit 2 (RPB2) were amplified using 983F/2218R and RPB2-5F/RPB2$7 \mathrm{Cr}$ primers according to van den Brink et al. (2012). DNA-directed RNA polymerase II largest subunit 1 (RPB1) was amplified with the primer pair CRPB1 and RPB1-Cr (Castlebury et al. 2004). The internal transcribed spacer (ITS) region was amplified using ITS4/ITS5 primers by PCR following the procedures described by White et al. (1990). PCR products were purified using the UNIQ-10 column PCR products purification kit [no. SK1141; Sangon Biotech (Shanghai) Co., Shanghai, China] in accordance with the manufacturer's protocol and sequenced at Sangon Biotech (Shanghai) Co. The resulting sequences were submitted to GenBank.

The new species Simplicillium cicadellidae, S. formicidae and S. lepidopterorum were registered in MycoBank with the numbers MB 831336, MB 831337 and MB 831335 , respectively.

\section{Sequence alignment and phylogenetic analyses}

DNA sequences generated in this study were assembled and edited using DNASTAR Lasergene software (version 6.0). Sequences of ITS, LSU, RPB1, RPB2 and TEF were selected based on previously published data by Nonaka et al. (2013), Zhang et al. (2017), Gomes et al. (2018), Crous et al. (2018) and Mongkolsamrit et al. (2018). Multiple sequence alignments for ITS, LSU, RPB1, RPB2 and TEF were carried out using MAFFT v7.037b (Katoh and Standley 2013). Sequence editing was performed with MEGA6 (Tamura et al. 2013), and the resulting output was in Fasta file format. The concatenated LSU+RPB1+RPB2+TEF and ITS+LSU sequences were assembled by SequenceMatrix v.1.7.8 (Vaidya et al. 2011). Gene concordance was assessed with the 'hompart' command in PAUP4.0b10 (Swofford 2002).

Two different analyses have been carried out using Bayesian inference (BI) and maximum likelihood (ML) methods. Analysis 1: To check the relationship between Simplicillium species and its allies in Cordycipitaceae based on the combined dataset of (LSU+RPB1+RPB2+TEF). Analysis 2: To check the relationship among Simplicillium spp. based on the combined dataset of (ITS+LSU). For the BI analysis, two runs were executed simultaneously for 10,000,000 generations, saving trees every 500 generations, with the GTR+G nucleotide substitution model across all the partitions, in MrBayes 3.2 (Ronquist et al. 2012). After the analysis was finished, each run was examined with the program Tracer v1.5 (Drummond and Rambaut 2007) to determine burn-in and confirm that both runs had converged. For the ML analysis in RAxML (Stamatakis 2014), the GTRGAMMA model was used for all the partitions in accordance with recommendations in the RAxML manual against the use of invariant sites. The final alignment is available from TreeBASE under submission ID: 24549 (http:// www.treebase.org) 


\section{Results}

\section{Phylogenetic analyses}

A phylogenetic tree of Simplicillium in Cordycipitaceae was generated from the maximum-likelihood (ML) and Bayesian inference (BI) based on a combined data set of LSU, RPB1, RPB2 and TEF sequence data. Statistical support ( $\geq 50 \% / 0.5)$ is shown at the nodes for ML bootstrap support/BI posterior probabilities (Fig. 1). The strain numbers are noted after each species' name. The tree is rooted with Purpureocillium lilacinum (Thom) Luangsa-ard, Houbraken, Hywel-Jones \& Samson (CBS 284.36 and CBS 431.87). The concatenated sequences including 40 taxa and contained 2,205 characters with gaps (LSU: 447, RPB1: 518, RPB2: 560, and TEF: 680).

A phylogenetic tree of Simplicillium species level was generated from the maximum-likelihood (ML) and Bayesian inference (BI) analysis based on a combined data set of ITS and LSU sequence data set. Statistical support $(\geq 50 \% / 0.5)$ are shown at the nodes for ML bootstrap support/BI posterior probabilities. The strain numbers are noted after each species' name. The tree is rooted with Pochonia chlamydosporia (Goddard) Zare \& W. Gams (CBS 103.65). The dataset includes 16 taxa and consists of 1,000 characters with gaps (ITS: 489 and LSU: 511).

Analysis 1: family Cordycipitaceae. The RAxML analysis of the combined dataset $(\mathrm{LSU}+\mathrm{RPB} 1+\mathrm{RPB} 2+\mathrm{TEF})$ yielded a best scoring tree (Fig. 1) with a final ML optimization likelihood value of $-24,337.973328$. Parameters for the GTR model of the concatenated data set was as follows: estimated base frequencies; $A=0.242689$, $\mathrm{C}=0.276532, \mathrm{G}=0.270879, \mathrm{~T}=0.209901$; substitution rates $\mathrm{AC}=0.926706$, $\mathrm{AG}=2.728719$, $\mathrm{AT}=0.823168, \mathrm{CG}=0.803225, \mathrm{CT}=6.257555, \mathrm{GT}=1.000000$; gamma distribution shape parameter $\alpha=0.410435$. The Bayesian analysis resulted in 20,001 trees after 10,000,000 generations. The first 4,000 trees, representing the burn-in phase of the analyses, were discarded, while the remaining 16,001 trees were used for calculating posterior probabilities in the majority rule consensus tree. In the phylogenetic tree (Fig. 1), Simplicillium cicadellidae, S. formicidae and S. lepidopterorum cluster with other Simplicillium species in a clade, and within the earliest diverging lineage in Cordycipitaceae.

Analysis 2: Simplicillium species. The RAxML analysis of the combined dataset (ITS+LSU) yielded a best scoring tree (Fig. 2) with a final ML optimization likelihood value of $-4,849.039588$. Parameters for the GTR model of the concatenated data set was as follows: Estimated base frequencies; $\mathrm{A}=0.243952, \mathrm{C}=0.258870$, $\mathrm{G}=0.268223, \mathrm{~T}=0.228956$; substitution rates $\mathrm{AC}=1.296760, \mathrm{AG}=2.678402$, $\mathrm{AT}=1.354112, \mathrm{CG}=1.488619, \mathrm{CT}=5.097242, \mathrm{GT}=1.000000$; gamma distribution shape parameter $\alpha=0.462419$. The Bayesian analysis resulted in 20,001 trees after 10,000,000 generations. The first 4,000 trees, representing the burn-in phase of the analyses, were discarded, while the remaining 16,001 trees were used for calculating posterior probabilities in the majority rule consensus tree. In the phylogenetic tree 


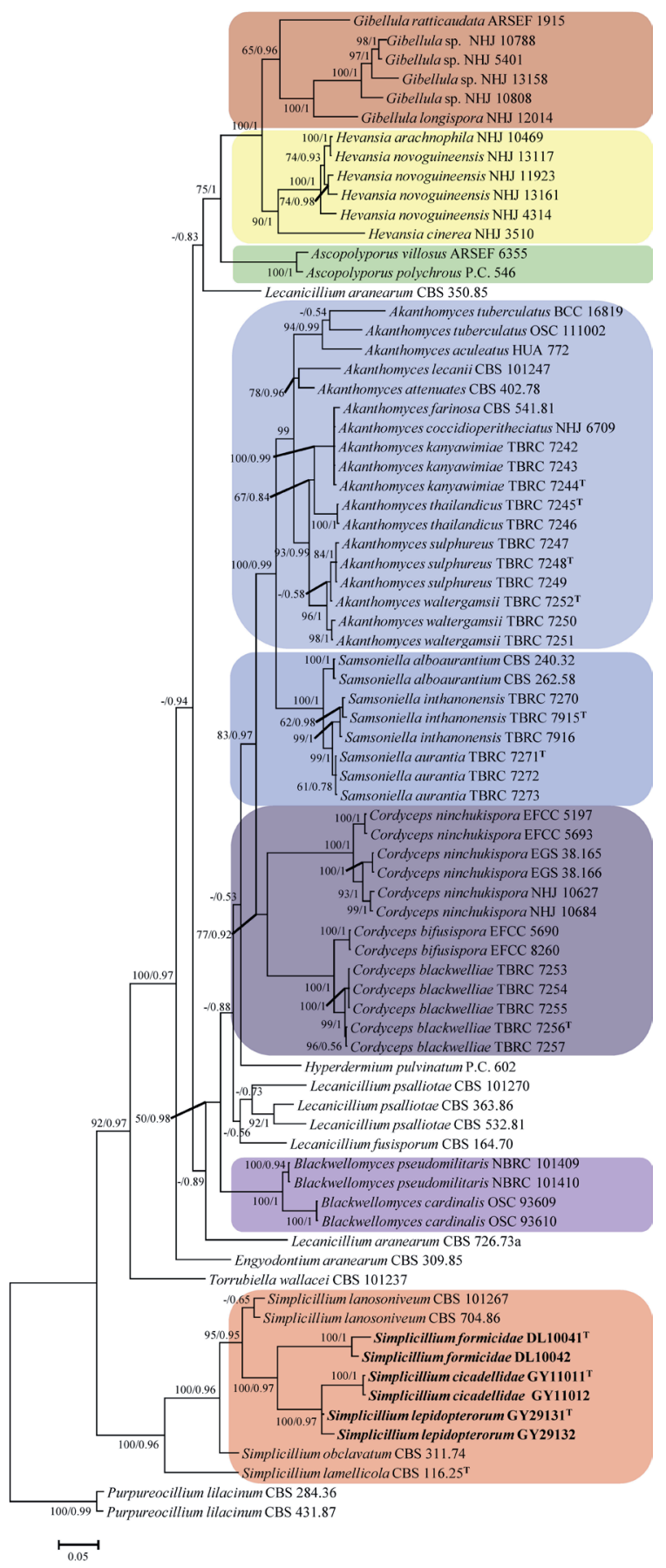

Figure I. Phylogenetic relationships among the genus Simplicillium and its allies in Cordycipitaceae based on multigene dataset (LSU, RPB1, RPB2 and TEF). Statistical support values $(\geq 0.5 / 50 \%)$ are shown at the nodes for ML bootstrap support/BI posterior probabilities. The tree is rooted with Purpureocillium lilacinum (CBS 284.36 and CBS 431.87). The new species are in bold face. T in the upper right corner indicates the type strains. 


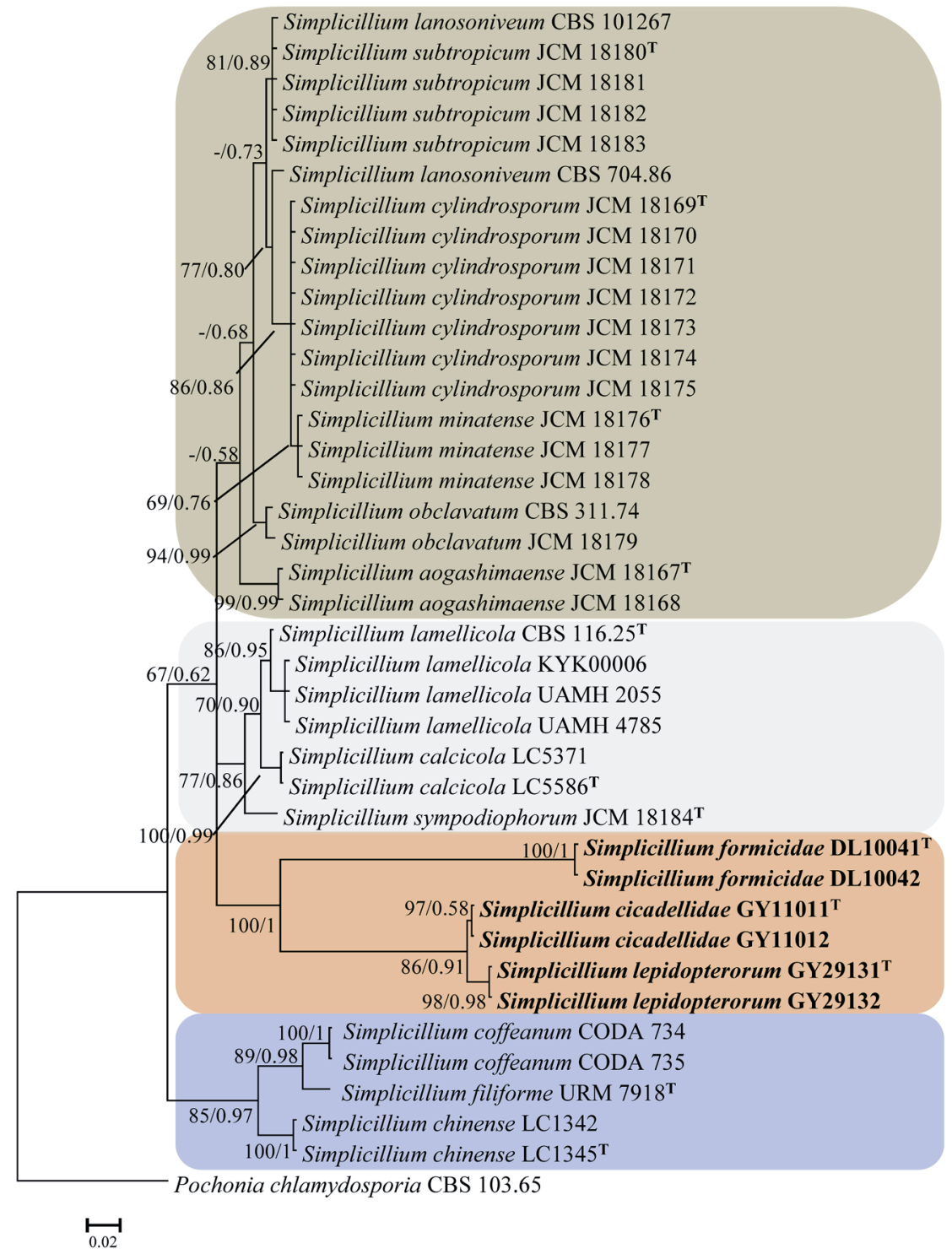

Figure 2. Phylogenetic relationships among the new taxa S. cicadellidae, S. formicidae, S. lepidopterorum and other Simplicillium species by ITS+LSU sequences. Statistical support values $(\geq 0.5 / 50 \%)$ are shown at the nodes for ML bootstrap support/BI posterior probabilities. The tree is rooted with Pochonia chlamydosporia (CBS 103.65). The new species are in bold face. $T$ in the upper right corner indicates the type strains.

(Fig. 2), Simplicillium species were resolved into four obvious clades. S. cicadellidae, $S$. formicidae and $S$. lepidopterorum were nested in a subclade and formed three independent branches, which received maximum statistical support (BI posterior probabilities 1, ML bootsrap 100\%). 
Table I. Taxa included in the phylogenetic analyses

\begin{tabular}{|c|c|c|c|c|c|c|}
\hline \multirow[t]{2}{*}{ Species } & \multirow[t]{2}{*}{ Strain No. } & \multicolumn{5}{|c|}{ GenBank Accession No. } \\
\hline & & ITS & LSU & RPB1 & RPB2 & TEF \\
\hline Akanthomyces aculeatus & HUA 772 & & KC519370 & & & KC519366 \\
\hline A. attenuates & CBS 402.78 & & AF339565 & EF468888 & EF468935 & EF468782 \\
\hline A. coccidioperitheciatus & NHJ 6709 & & EU369042 & EU369067 & EU369086 & EU369025 \\
\hline A. farinosa & CBS 541.81 & & & & & JQ425686 \\
\hline \multirow[t]{3}{*}{ A. kanyawimiae } & TBRC 7242 & & MF140718 & MF140784 & MF140808 & MF140838 \\
\hline & TBRC 7243 & & MF140717 & MF140783 & MF140807 & MF140837 \\
\hline & TBRC 7244 & & MF140716 & & & MF140836 \\
\hline A. lecanii & CBS 101247 & & AF339555 & DQ522407 & DQ522466 & DQ522359 \\
\hline \multirow[t]{3}{*}{ A. sulphureus } & TBRC 7247 & & MF140720 & & & MF140841 \\
\hline & TBRC 7248 & & MF140722 & MF140787 & MF140812 & MF140843 \\
\hline & TBRC 7249 & & MF140721 & MF140786 & MF140734 & MF140842 \\
\hline \multirow[t]{2}{*}{ A. thailandicus } & TBRC 7245 & & & & MF140809 & MF140839 \\
\hline & TBRC 7246 & & MF140719 & & MF140810 & MF140840 \\
\hline \multirow[t]{2}{*}{ A. tuberculatus } & BCC 16819 & & GQ249987 & & & GQ250037 \\
\hline & OSC 111002 & & DQ518767 & DQ522384 & DQ522435 & DQ522338 \\
\hline \multirow[t]{3}{*}{ A. waltergamsii } & TBRC 7250 & & MF140715 & & & MF140835 \\
\hline & TBRC 7251 & & MF140713 & MF140781 & MF140805 & MF140833 \\
\hline & TBRC 7252 & & MF140714 & MF140782 & MF140806 & MF140834 \\
\hline Ascopolyporus polychrous & P.C. 546 & & DQ118737 & DQ127236 & & DQ118745 \\
\hline A. villosus & ARSEF 6355 & & AY886544 & DQ127241 & & DQ118750 \\
\hline \multirow[t]{2}{*}{ Blackwellomyces cardinalis } & OSC 93609 & & AY184962 & DQ522370 & DQ522422 & DQ522325 \\
\hline & OSC 93610 & & AY184963 & EF469088 & EF469106 & EF469059 \\
\hline \multirow[t]{2}{*}{ B. pseudomilitaris } & NBRC 101409 & & JN941393 & JN992482 & & \\
\hline & NBRC 101410 & & JN941394 & JN992481 & & \\
\hline \multirow[t]{2}{*}{ Cordyceps bifusispora } & EFCC 5690 & & EF468806 & EF468854 & EF468909 & EF468746 \\
\hline & EFCC 8260 & & EF468807 & EF468855 & EF468910 & EF468747 \\
\hline \multirow[t]{5}{*}{ C. blackwelliae } & TBRC 7253 & & MF140705 & MF140774 & MF140798 & MF140825 \\
\hline & TBRC 7254 & & MF140704 & MF140773 & MF140797 & MF140824 \\
\hline & TBRC 7255 & & MF140703 & MF140772 & MF140796 & MF140823 \\
\hline & TBRC 7256 & & MF140702 & MF140771 & MF140795 & MF140822 \\
\hline & TBRC 7257 & & MF140701 & MF140770 & MF140794 & MF140821 \\
\hline \multirow[t]{6}{*}{ C. ninchukispora } & EFCC 5197 & & EF468820 & EF468868 & & EF468760 \\
\hline & EFCC 5693 & & EF468821 & EF468869 & & EF468762 \\
\hline & EGS 38.165 & & EF468846 & EF468900 & & EF468795 \\
\hline & EGS 38.166 & & EF468847 & EF468901 & & EF468794 \\
\hline & NHJ 10627 & & EF468822 & EF468870 & & EF468763 \\
\hline & NHJ 10684 & & EF468823 & EF468871 & & EF468761 \\
\hline Engyodontium aranearum & CBS 309.85 & & AF339526 & DQ522387 & DQ522439 & DQ522341 \\
\hline Gibellula longispora & NHJ 12014 & & & EU369055 & EU369075 & EU369017 \\
\hline G. pulchra & NHJ 10808 & & EU369035 & EU369056 & EU369076 & EU369018 \\
\hline G. ratticaudata & ARSEF 1915 & & DQ518777 & DQ522408 & DQ522467 & DQ522360 \\
\hline \multirow[t]{3}{*}{ Gibellula sp. } & NHJ 5401 & & & EU369059 & EU369079 & \\
\hline & NHJ 10788 & & EU369036 & EU369058 & EU369078 & EU369019 \\
\hline & NHJ 13158 & & EU369037 & EU369057 & EU369077 & EU369020 \\
\hline Hevansia arachnophila & NHJ 10469 & & EU369031 & EU369047 & & EU369008 \\
\hline H. cinerea & NHJ 3510 & & & EU369048 & EU369070 & EU369009 \\
\hline \multirow[t]{4}{*}{ H. novoguineensis } & NHJ 4314 & & & EU369051 & EU369071 & EU369012 \\
\hline & NHJ 11923 & & EU369032 & EU369052 & EU369072 & EU369013 \\
\hline & NHJ 13117 & & & EU369049 & EU369073 & EU369010 \\
\hline & NHJ 13161 & & & EU369050 & & EU369011 \\
\hline Hyperdermium pulvinatum & P.C. 602 & & AF242353 & DQ127237 & & DQ118746 \\
\hline L. aranearum & CBS 726.73a & & AF339537 & EF468887 & EF468934 & EF468781 \\
\hline L. fusisporum & CBS $164.70 \mathbf{T}$ & & AF339549 & EF468889 & & EF468783 \\
\hline
\end{tabular}




\begin{tabular}{|c|c|c|c|c|c|c|}
\hline \multirow[t]{2}{*}{ Species } & \multirow[t]{2}{*}{ Strain No. } & \multicolumn{5}{|c|}{ GenBank Accession No. } \\
\hline & & ITS & LSU & RPB1 & RPB2 & TEF \\
\hline \multirow[t]{3}{*}{ L. psalliotae } & CBS 363.86T & & AF339559 & EF468890 & & EF468784 \\
\hline & CBS 532.81 & & AF339560 & EF469096 & EF469112 & EF469067 \\
\hline & CBS 101270 & & EF469081 & EF469095 & EF469113 & EF469066 \\
\hline Pochonia chlamydosporia & CBS 103.65 & MH858504 & & & & \\
\hline \multirow[t]{2}{*}{ Purpureocillium lilacinum } & CBS 284.36 & & FR775484 & EF468898 & EF468941 & EF468792 \\
\hline & CBS 431.87 & & EF468844 & EF468897 & $\mathrm{EF} 468940$ & EF468791 \\
\hline \multirow[t]{2}{*}{ Samsoniella alboaurantium } & CBS 240.32 & & JF415979 & JN049895 & JF415999 & JF416019 \\
\hline & CBS 262.58 & & MG665232 & & & JQ425685 \\
\hline \multirow[t]{3}{*}{ S. aurantia } & TBRC 7271T & & MF140728 & MF140791 & MF140818 & MF140846 \\
\hline & TBRC 7272 & & MF140727 & MF140817 & & MF140845 \\
\hline & TBRC 7273 & & MF140726 & & MF140816 & MF140844 \\
\hline \multirow[t]{3}{*}{ S. inthanonensis } & TBRC 7915T & & MF140725 & MF140790 & MF140815 & MF140849 \\
\hline & TBRC 7916 & & MF140724 & MF140789 & MF140814 & MF140848 \\
\hline & TBRC 7270 & & MF140723 & MF140788 & MF140813 & MF140847 \\
\hline Simplicillium & JCM 18167T & AB604002 & & & & \\
\hline aogashimaense & JCM 18168 & AB604004 & & & & \\
\hline \multirow[t]{2}{*}{ S. calcicola } & LC 5371 & KU746705 & KU74675 & & & \\
\hline & LC 5586T & KU746706 & KU746752 & & & \\
\hline \multirow[t]{2}{*}{ S. chinense } & LC 1342 & JQ410323 & JQ410321 & & & \\
\hline & LC 1345 & NR155782 & JQ410322 & & & \\
\hline \multirow[t]{2}{*}{ S. cicadellidae } & GY11011T & MN006243 & MN006249 & MN022271 & & MN022263 \\
\hline & GY11012 & MN006244 & MN006250 & MN022272 & & MN022264 \\
\hline \multirow[t]{2}{*}{ S. coffeanum } & COAD 2057T & MF066034 & MF066032 & & & \\
\hline & COAD 2061 & MF066035 & MF066033 & & & \\
\hline \multirow[t]{7}{*}{ S. cylindrosporum } & JCM 18169T & AB603989 & & & & \\
\hline & JCM 18170 & AB603994 & & & & \\
\hline & JCM 18171 & AB603997 & & & & \\
\hline & JCM 18172 & AB603998 & & & & \\
\hline & JCM 18173 & AB603999 & & & & \\
\hline & JCM 18174 & $\mathrm{AB} 604005$ & & & & \\
\hline & JCM 18175 & AB604006 & & & & \\
\hline S. filiforme & URM 7918 & MH979338 & MH979399 & & & \\
\hline \multirow{2}{*}{ S. formicidae } & DL10041T & MN006241 & MN006247 & MN022269 & MN022267 & \\
\hline & DL10042 & MN006242 & MN006248 & MN022270 & MN022268 & \\
\hline \multirow[t]{3}{*}{ S. lamellicola } & CBS 116.25T & AJ292393 & AF339552 & DQ522404 & DQ522462 & DQ522356 \\
\hline & UAMH 2055 & AF108471 & & & & \\
\hline & UAMH 4785 & AF108480 & & & & \\
\hline S. lamellicola ${ }^{\mathrm{b}}$ & KYK00006 & AB378533 & & & & \\
\hline \multirow[t]{2}{*}{ S. lanosoniveum } & CBS 704.86 & AJ292396 & AF339553 & DQ522406 & DQ522464 & DQ522358 \\
\hline & CBS 101267 & AJ292395 & AF339554 & DQ522405 & DQ522463 & DQ522357 \\
\hline \multirow[t]{2}{*}{ S. lepidopterorum } & GY29131T & MN006246 & MN006251 & MN022273 & & MN022265 \\
\hline & GY29132 & MN006245 & MN006252 & MN022274 & & MN022266 \\
\hline \multirow[t]{3}{*}{ S. minatense } & JCM 18176T & AB603992 & & & & \\
\hline & JCM 18177 & AB603991 & & & & \\
\hline & JCM 18178 & AB603993 & & & & \\
\hline S. obclavatum & CBS $311.74 \mathbf{T}$ & AJ292394 & AF339517 & & & EF468798 \\
\hline & JCM 18179 & AB604000 & & & & \\
\hline S. subtropicum & JCM 18180T & AB603990 & & & & \\
\hline & JCM 18181 & AB603995 & & & & \\
\hline & JCM 18182 & AB603996 & & & & \\
\hline & JCM 18183 & AB604001 & & & & \\
\hline S. sympodiophorum & JCM 18184T & AB604003 & & & & \\
\hline Torrubiella wallacei & CBS $101237 \mathbf{T}$ & & AY184967 & EF469102 & EF469119 & EF469073 \\
\hline
\end{tabular}

$\mathbf{T}=$ type strains, strain and sequences generated in this study are shown in bold. 


\section{Taxonomy}

Simplicillium cicadellidae W.H. Chen, C. Liu, Y.F. Han, J.D. Liang, Z.Q. Liang sp. nov.

MycoBank: MB 831336

Figure 3

Etymology. The epithet cicadellidae refers to an insect host in family Cicadellidea.

Diagnosis. Characterized by phialides always solitary and rather long and narrow, $12.9-18.3 \times 0.8-1.1 \mu \mathrm{m}$. Conidia adhering in globose slimy heads, mostly ellipsoidal,
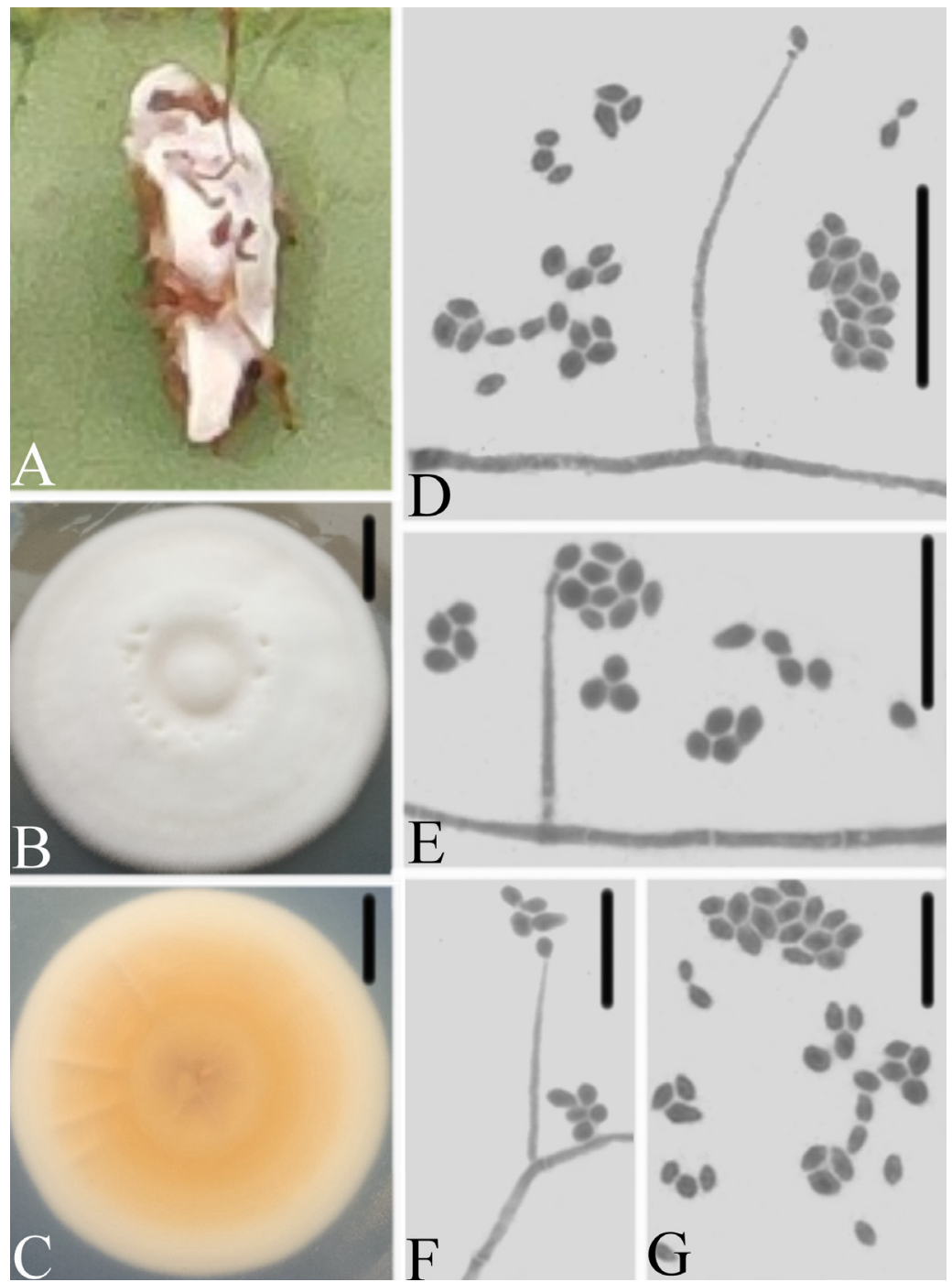

Figure 3. Simplicillium cicadellidae $\mathbf{A}$ infected leafhopper (Hemiptera) B-C culture plate, showing the front $(\mathbf{B})$ and the reverse $(\mathbf{C})$ of the colony, cultured on PDA medium D-F phialides solitary, conidia adhering ellipsoidal slimy head and conidia $\mathbf{G}$ conidia. Scale bars: $10 \mathrm{~mm}(\mathbf{B}, \mathbf{C}), 10 \mu \mathrm{m}(\mathbf{D}, \mathbf{E}, \mathbf{F}, \mathbf{G})$. 
$1.8-2.8 \times 1.4-1.8 \mu \mathrm{m}$. Octahedral crystals absent. Reverse of colony yellowish, especially in the middle, and radially sulcate.

Type. CHINA, Guizhou Province, Huaxi District (26²3'25.92"N, 106²1'3.35"E), 9 November 2018, Wanhao Chen, holotype GZAC GY1101, extype culture GZAC GY11011. Sequences from isolated strain GY11011 has been deposited in GenBank with accession numbers: ITS = MN006243, LSU = MN006249, $\mathrm{RPB1}=\mathrm{MN022271}$ and TEF $=$ MN022263.

Description. Colonies reaching $45-47 \mathrm{~mm}$ in diameter in $14 \mathrm{~d}$ on PDA; white; reverse yellowish, especially in the middle, and radially sulcate. Hyphae septate, hyaline, smooth-walled, 0.9-1.9 $\mu \mathrm{m}$ wide. Phialides arising from aerial hyphae, gradually tapering towards apex, without basal septa, always solitary and rather long and narrow, $12.9-18.3 \times 0.8-1.1 \mu \mathrm{m}$. Conidia adhering in ellipsoidal slimy heads, mostly ellipsoidal, hyaline, smooth-walled, $1.8-2.8 \times 1.4-1.8 \mu \mathrm{m}$. Octahedral crystals absent.

Host. Leafhopper (Hemiptera)

Distribution. Huaxi District, Guizhou Province, China

Remarks. Zare and Gams (2001) summarized the typical characteristics of Simplicillium as having mostly solitary phialides arising from aerial hyphae, conidia adhering in globose slimy heads or imbricate chains, crystals commonly present, fungicolous and on various other substrata. Simplicillium cicadellidae was easily identified as belonging to Simplicillium because of its solitary phialides, conidia adhering in ellipsoidal slimy heads, and lack of octahedral crystals. Comparing with the typical characteristics of 12 species (Table 2), it was easily distinguished from other species in having the phialides always solitary and rather long and narrow $(12.9-18.3 \times 0.8-1.1 \mu \mathrm{m})$, the conidia adhering in globose slimy heads, which are mostly ellipsoidal (1.8-2.8 $\times 1.4-1.8$ $\mu \mathrm{m})$, and the octahedral crystals absent. The reverse of colony was yellowish, especially in the middle, and radially sulcate. Based on ITS and LSU rDNA, S. cicadellidae is phylogenetically close to $S$. formicidae and S. lepidopterorum. However, S. cicadellidae has ellipsoidal conidia and shorter phialides $(12.9-18.3 \times 0.8-1.1 \mu \mathrm{m})$, and the reverse of colony was yellowish.

Simplicillium formicidae W.H. Chen, C. Liu, Y.F. Han, J.D. Liang, Z.Q. Liang, sp. nov. MycoBank: MB 831337

Figure 4

Etymology. The epithet formicidae refers to an insect host in family Formicidae.

Diagnosis. Characterized by phialides always being solitary and rather long and narrow, 51-70.1 $\times$ 0.7-0.9 $\mu \mathrm{m}$. Conidia adhering in globose slimy heads, mostly filiform to fusoid, 3.9-7.9 $\times 0.8-1.3 \mu \mathrm{m}$. Octahedral crystals absent.

Type. CHINA, Guizhou Province, Rongjiang County (2601'58.70"N, 108²4'48.06"E), 1 October 2018, Wanhao Chen, holotype GZAC DL1004, ex-type culture GZAC DL10041. Sequences from isolated strain DL10041 has been deposited in GenBank with accession numbers: ITS = MN006241, LSU = MN006247, RPB1 = MN022269 and RPB2 = MN022267. 


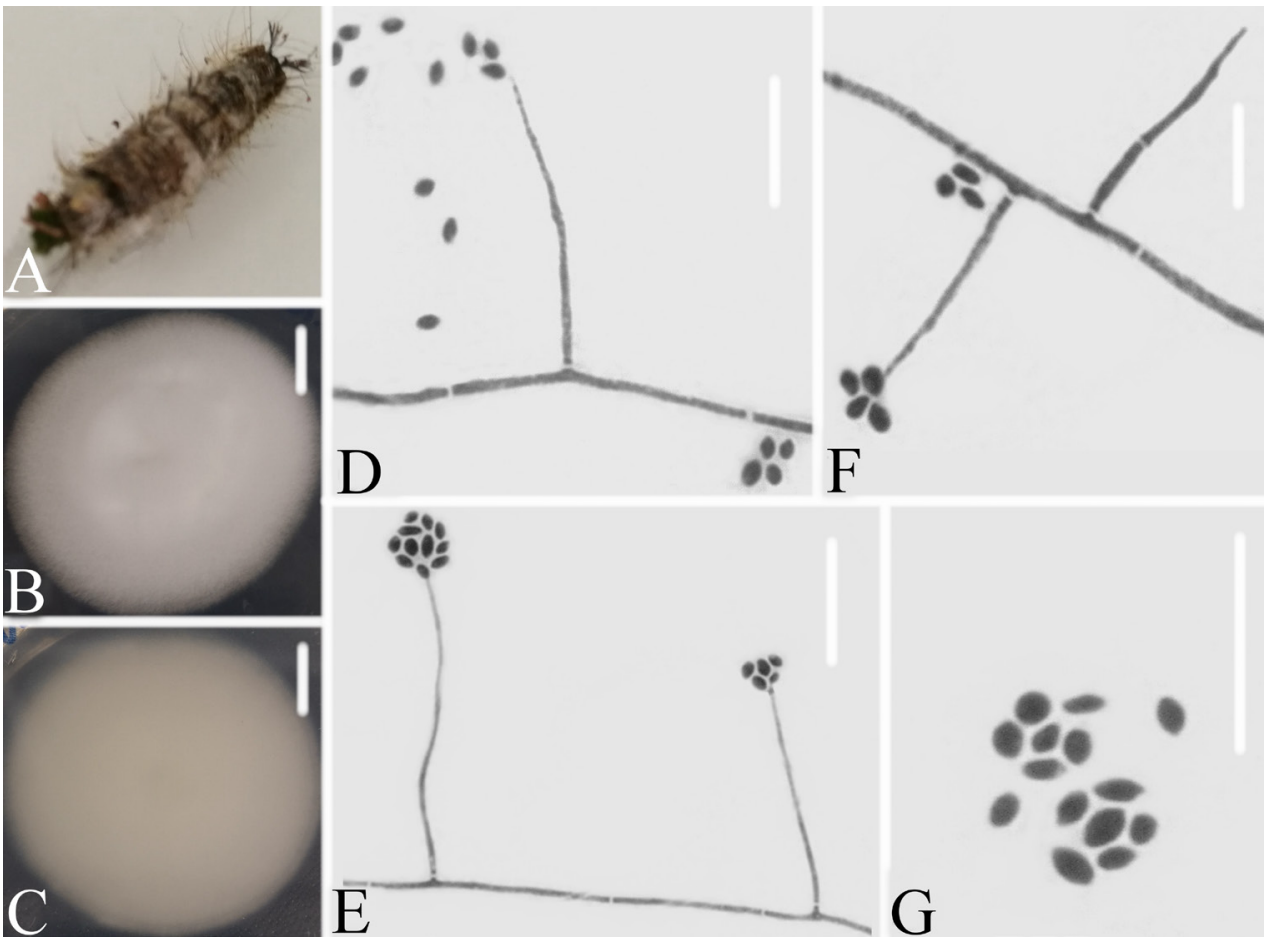

Figure 4. Simplicillium lepidopterorum $\mathbf{A}$ infected carpenterworm (Lepidoptera) B, C culture plate, showing the front $(\mathbf{B})$ and the reverse $(\mathbf{C})$ of the colony, cultured on PDA medium $\mathbf{D}, \mathbf{E}, \mathbf{F}$ phialides solitary and conidia in globose heads $\mathbf{D}$ conidia. Scale bars: $10 \mathrm{~mm}(\mathbf{B}, \mathbf{C}), 10 \mu \mathrm{m}(\mathbf{D}, \mathbf{E}, \mathbf{F}, \mathbf{G})$.

Description. Colonies reaching $26-32 \mathrm{~mm}$ in diameter in $14 \mathrm{~d}$ on PDA; white; reverse pale brown to brown, and with brown secretions. Hyphae septate, hyaline, smooth-walled, $1.2-1.8 \mu \mathrm{m}$ wide. Phialides arising from aerial hyphae, gradually tapering towards the apex, without basal septa, always solitary and rather long and narrow, 51-70.1 × 0.7-0.9 $\mu \mathrm{m}$. Conidia adhering in globose slimy heads, mostly filiform to fusoid, hyaline, smooth-walled, 3.9-7.9 $\times 0.8-1.3 \mu \mathrm{m}$. Octahedral crystals absent.

\section{Host. Ant (Hymenoptera)}

Distribution. Rongjiang County, Guizhou Province, China

Remarks. Simplicillium formicidae was easily identified as belonging to Simplicillium because of its solitary phialides, conidia adhering in globose slimy heads, and lack of octahedral crystals. Compared with the typical characteristics of 12 species (Table 2 ), it was easily distinguished from those species by having the phialides always solitary and rather long and narrow $(51-70.1 \times 0.7-0.9 \mu \mathrm{m})$ and the conidia mostly filiform to fusoid $(3.9-7.9 \times 0.8-1.3 \mu \mathrm{m})$, and adhering in globose slimy heads, and in having octahedral crystals absent. Based on ITS and LSU rDNA, S. formicidae is phylogenetically close to $S$. cicadellidae and S. lepidopterorum. However, S. formicidae has larger filiform to fusoid conidia $(3.9-7.9 \times 0.8-1.3 \mu \mathrm{m})$. 
Simplicillium lepidopterorum W.H. Chen, C. Liu, Y.F. Han, J.D. Liang \& Z.Q. Liang, sp. nov.

MycoBank: MB 831335

Figure 5

Etymology. The epithet lepidopterorum refers to an insect host in order Lepidoptera.

Diagnosis. Characterized by phialides always being solitary and rather long and narrow, 15.3-26.2 $\times 0.7-1.4 \mu \mathrm{m}$, Conidia adhering in globose slimy heads, mostly ellipsoidal, 1.6-2.4 × 1.4-1.7 $\mu \mathrm{m}$. Octahedral crystals absent. The reverse of colony was pale white.

Type. CHINA, Guizhou Province, Huaxi District $\left(26^{\circ} 23^{\prime} 25.92 " \mathrm{~N}\right.$, $106^{\circ} 41^{\prime} 3.35^{\prime \prime E}$ ), 31 July 2018, Wanhao Chen, holotype GZAC GY2913, ex-type culture GZAC GY29131, sequences from isolated strain GY29131 has been deposited in GenBank with accession numbers: ITS $=$ MN006246, LSU $=$ MN006251, RPB1 = MN022273 and TEF = MN022265.

Description. Colonies reaching $48-51 \mathrm{~mm}$ in diameter in $14 \mathrm{~d}$ on PDA; white; reverse pale white. Hyphae septate, hyaline, smooth-walled, 1.1-2.2 $\mu$ m wide. Phi-

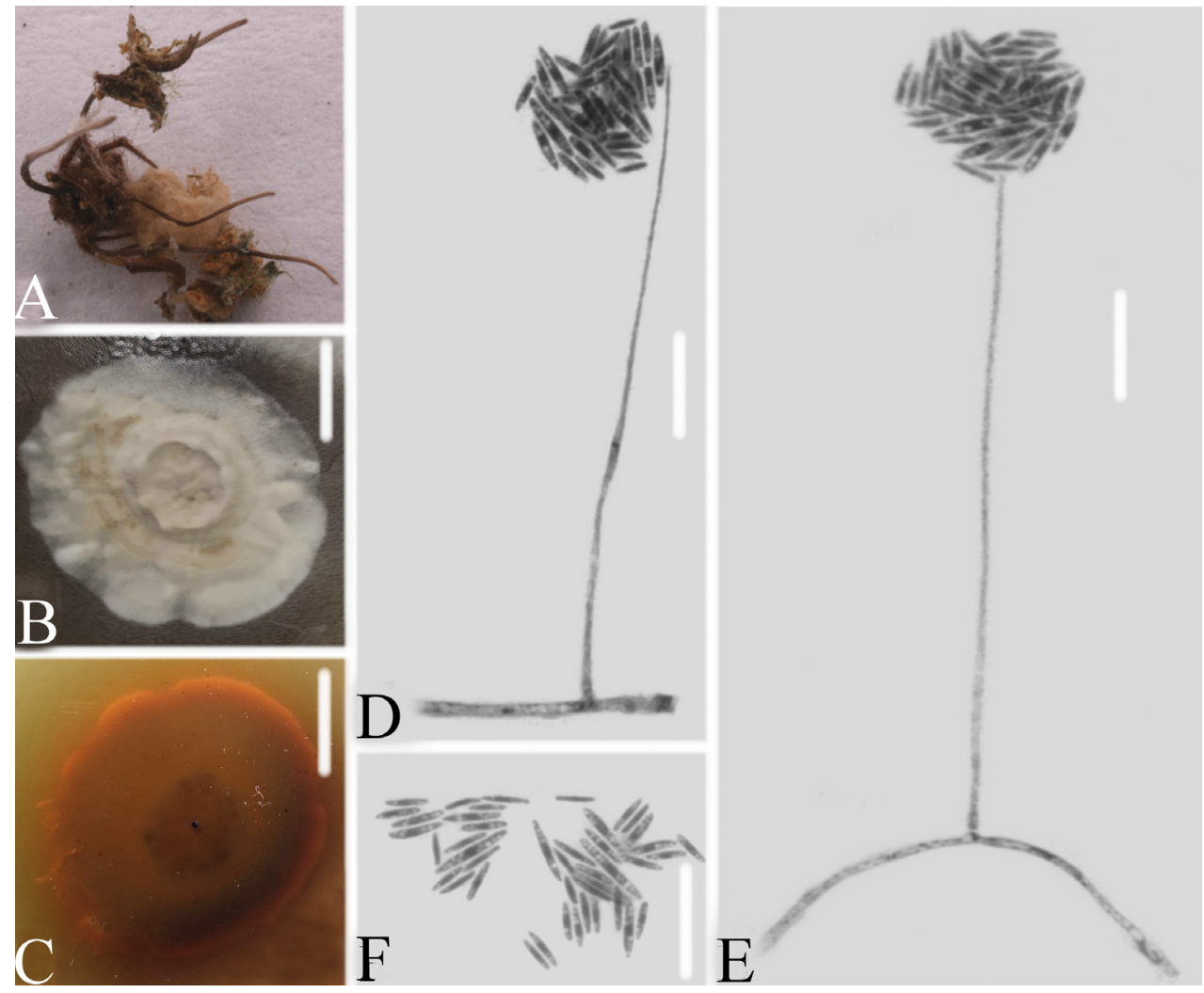

Figure 5. Simplicillium formicidae $\mathbf{A}$ isolated substrate an infected ant (Hymenoptera) B-C culture plate, showing the front $(\mathbf{B})$ and the reverse $(\mathbf{C})$ of the colony, cultured on PDA medium $\mathbf{D}, \mathbf{E}$ phialides solitary, conidia adhering globose slimy head and conidia $\mathbf{F}$ conidia. Scale bars: $10 \mathrm{~mm}(\mathbf{B}, \mathbf{C}), 10 \mu \mathrm{m}(\mathbf{D}, \mathbf{E}, \mathbf{F})$. 
Table 2. Morphological comparison of three new species with other Simplicillium species

\begin{tabular}{|c|c|c|c|c|c|}
\hline \multirow[t]{2}{*}{ Species } & \multicolumn{4}{|c|}{ Morphological characteristics } & \multirow[t]{2}{*}{ Notes } \\
\hline & \begin{tabular}{|c|} 
Phialide (Conidiogenous \\
cell) $(\mu \mathrm{m})$
\end{tabular} & Conidia $(\mu \mathrm{m})$ & Conidia mass & $\begin{array}{c}\text { Octahedral } \\
\text { crystals }\end{array}$ & \\
\hline S. aogashimaense $e^{2}$ & $(19-) 23-53 \times 1.2-2.0$ & cylindrical, $4.2-6.5 \times 1.2-2.0$ & globose heads & present & $\begin{array}{c}\text { Chlamydospores } \\
\text { present }\end{array}$ \\
\hline S. calcicola $^{\mathrm{b}}$ & $14-38 \times 1-2$ & $\begin{array}{c}\text { micro-: globose, oval or } \\
\text { ellipsoidal, } 2-3.5 \times 1-1.5 \\
\text { macro-: fusiform, } 4.5-8 \times 1-2\end{array}$ & & absent & \\
\hline S. chinense $^{c}$ & $(6.0-) 15-30(-68.0) \times 1.5$ & $\begin{array}{l}\text { oval, ellipsoidal or cylindrical } \\
3.5-5.0 \times 1.0-1.5\end{array}$ & $\begin{array}{c}\text { branched or } \\
\text { unbranched chains }\end{array}$ & present & \\
\hline S. coffeanum ${ }^{\mathrm{d}}$ & $11-40(-70) \times 1.0-2.4$ & $\begin{array}{c}\text { micro-: spindle-shaped, } \\
5.3-8.8 \times 1.0-1.6 \\
\text { macro-: ellipsoidal to fusiform, } \\
2.2-3.8 \times 0.8-1.5\end{array}$ & $\begin{array}{l}\text { subglobose to } \\
\text { ellipsoidal heads }\end{array}$ & absent & \\
\hline S. cylindrosporum ${ }^{\mathrm{a}}$ & $17-32 \times 1.2-2.0(-2.5)$ & $\begin{array}{c}\text { cylindrical, } 3.0-4.5(-5.0) \times \\
1.0-2.0\end{array}$ & globose heads & present & \\
\hline S. filiforme $e^{\mathrm{e}}$ & $9-18 \times 1$ & fusoid to filiform, $7.2-12.5 \times 1$ & zigzag chains & absent & \\
\hline S. lamellicola $^{\mathrm{f}}$ & $15-50 \times 0.7-1.0$ & $\begin{array}{l}\text { micro-: spindle-shaped, } \\
4.5-9.0 \times 0.8-1.2 \\
\text { macro-: oval to ellipsoidal, } \\
2.0-3.0 \times 0.7-1.2\end{array}$ & $\begin{array}{l}\text { subglobose to } \\
\text { ellipsoidal heads }\end{array}$ & present & \\
\hline S. lanosoniveum ${ }^{\mathrm{f}}$ & $15-35 \times 0.7-1.5$ & $\begin{array}{c}\text { subglobose, oval, ellipsoidal } \\
1.5-3 \times 0.7-1.3\end{array}$ & globose heads & present & \\
\hline S. minatense ${ }^{2}$ & $11-31(-47) \times 1.0-1.7$ & $\begin{array}{c}\text { globose to subglobose, } \\
\text { sometimes ellipsoidal, } 2.0-3.5 \\
\times 1.8-2.5(-2.8)\end{array}$ & globose heads & present & \\
\hline S. obclavatum ${ }^{\mathrm{f}}$ & $30-52 \times 0.8-1.2$ & $\begin{array}{c}\text { obclavate to ellipsoidal, } \\
2.5-3.5 \times 1-2\end{array}$ & $\begin{array}{l}\text { short imbricate } \\
\text { chains }\end{array}$ & present & \\
\hline S. subtropicum ${ }^{\mathrm{a}}$ & $\begin{array}{c}(15-) 20-42(-50) \times \\
1.0-2.3\end{array}$ & $\begin{array}{l}\text { subglobose to ellipsoidal, } \\
2.3-4.0(-4.5) \times 1.5-3.3\end{array}$ & globose heads & present & \\
\hline $\begin{array}{l}\text { S. } \\
\text { sympodiophorum }\end{array}$ & $\begin{array}{l}20-34(-47) \times 0.5-1.3 \\
\text { denticles present }\end{array}$ & $\begin{array}{l}\text { oval to ellipsoidal, } 2.2-3.5 \times \\
1.0-2.0\end{array}$ & & present & \\
\hline S. cicadellidae & $12.9-18.3 \times 0.8-1.1$ & ellipsoidal, $1.8-2.8 \times 1.4-1.8$ & ellipsoidal heads & absent & $\begin{array}{c}\text { colonies reverse } \\
\text { pale white }\end{array}$ \\
\hline S. formicidae & $51-70.1 \times 0.7-0.9$ & $\begin{array}{l}\text { filiform to fusoid, } 3.9-7.9 \times \\
0.8-1.3\end{array}$ & globose heads & absent & \\
\hline S. lepidopterorum & $15.3-26.2 \times 0.7-1.4$ & ellipsoidal, 1.6-2.4 × 1.4-1.7 & globose heads & absent & $\begin{array}{c}\text { colonies reverse } \\
\text { yellowish }\end{array}$ \\
\hline
\end{tabular}

a-f: data are derived from Zare and Gams (2001), Nonaka et al. (2013), Zhang et al. (2017), Liu and Cai 2012, Gomes et al. (2018) and Crous et al. (2018), respectively.

alides arising from aerial hyphae, gradually tapering towards the apex, without basal septa, always solitary and rather long and narrow, 15.3-26.2 $\times 0.7-1.4 \mu \mathrm{m}$. Conidia adhering in globose slimy heads, ellipsoidal to fusiform, hyaline, smooth-walled, 1.6$2.4 \times 1.4-1.7 \mu \mathrm{m}$. Octahedral crystals absent.

\section{Host. Carpenter worm (Lepidoptera)}

Distribution. Huaxi District, Guizhou Province, China

Remarks. Simplicillium lepidopterorum was easily identified as belonging to Simplicillium because of its solitary phialides, conidia adhering in globose slimy heads, and lack of octahedral crystals. Comparing with the typical characteristics of 12 species (Table 2), S. lepidopterorum could easily distinguished from other species by having the phialides always solitary and rather long and narrow, 15.3-26.2 $\times 0.7-1.4 \mu \mathrm{m}$. Conidia ellipsoidal $(1.6-2.4 \times 1.4-1.7 \mu \mathrm{m})$, adhering in globose slimy heads, and in 
having the octahedral crystals absent. Based on ITS and LSU rDNA, S. lepidopterorum is phylogenetically close to $S$. cicadellidae and $S$. formicidae. However, S. lepidopterorum has ellipsoidal conidia, longer phialides $(15.3-26.2 \times 0.7-1.4 \mu \mathrm{m})$, and the reverse of colony was pale white.

Key

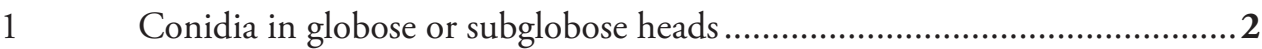

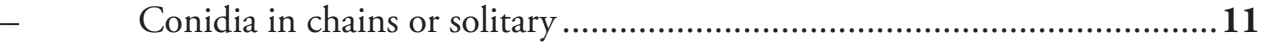

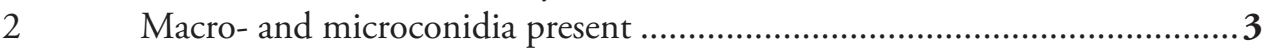

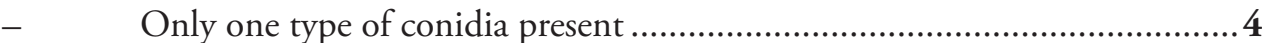

3 Octahedral crystals present.................................................... S. lamellicola

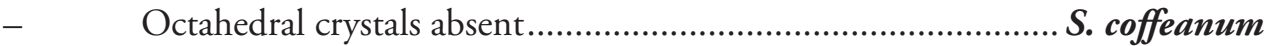

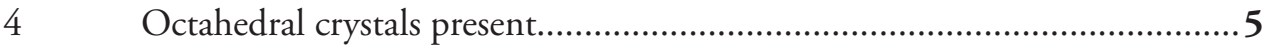

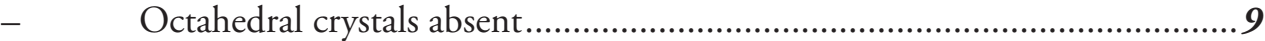

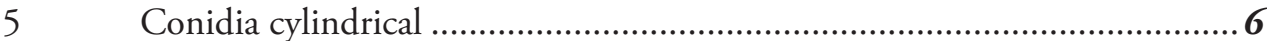

- $\quad$ Conidia subglobose or ellipsoidal......................................................... 7

6 Chlamydospores present, conidia 4.2-6.5 × 1.2-2.0 $\mu \mathrm{m} . .$. S. aogashimaense

- $\quad$ Chlamydospores absent, conidia 3.0-4.5 (-5.0) × 1.0-2.0 $\mu \mathrm{m}$

S. cylindrosporum

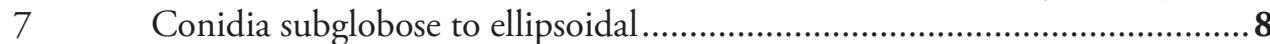

- Conidia oval or ellipsoidal to subcylindrical, 1.5-3.0 × 0.7-1.3 $\mu \mathrm{m}$.

S. lanosoniveum

8 Conidia subglobose to ellipsoidal, 2.3-4.0 (-4.5) × 1.5-3.3 $\mu \mathrm{m}$

S. subtropicum

- $\quad$ Conidia globose to subglobose, sometimes ellipsoidal, $2.5-3.5 \times 1.8-2.5$ $(-2.8) \mu \mathrm{m}$

S. minatense

9 Conidia ellipsoidal 10

Conidia filiform to fusoid

S. formicidae

10 The reverse of colony pale white, phialide $12.9-18.3 \times 0.8-1.1 \mu \mathrm{m}$

S. cicadellidae

The reverse of colony yellowish, phialide 15.3-26.2 × 0.7-1.4 $\mu \mathrm{m}$

S. lepidopterorum

11 Denticles present in conidiogenous cell (phialide).........S. sympodiophorum

- Denticles absent in conidiogenous cell (phialide) .....................................12

12 Macro- and microconidia present …………............................. S. calcicola

Only one type of conidia present ............................................................ 13

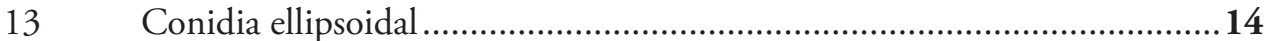

Conidia fusoid to filiform, form zigzag chains.

S. filiforme

14 Conidia in branched or unbranched chains, 3.5-5.0 $\times 1.0-1.5 \mu \mathrm{m}$. S. chinense Conidia in short imbricate chains, $2.5-3.5 \times 1.0-2.0 \mu \mathrm{m} . . . . . S$. obclavatum 


\section{Discussion}

Two types of the evolutionary correlation patterns between fungi and hosts are known, co-evolutionary patterns and the more frequent host jump events (Spatafora et al. 2007). The generation of host jumping is closely related to a common living environment (Vega et al. 2009). Nutritional sources are very important factors in determining whether a host has undergone a host jump. The nutritional model of Hypocreales fungi is from plants (including living plants and plant residues) to animals (especially insects), and finally to fungi. Plants and their residues were the initial sources of nutrition for the common ancestor of Hypocreaceae and Clavicipitaceae. The jumps from plants to animals and then to fungi indicate that the fungal nutrient requirements have changed with the environment (Spatafora et al. 2007). Prediction of the characteristics and evolutionary placement of any given member should be based on the correlation between molecular-phylogenetic genealogy and nutritional preferences (Spatafora et al. 2007; Vega et al. 2009). Additionally, host insect species are an important diagnostic feature in the identification of entomopathogenic fungi.

Among the 12 reported Simplicillium species, S. aogashimaense (soil), S. calcicola (calcareous rock), S. chinense (decaying wood), S. cylindrosporum (soil), S. minatense (soil), S. obclavatum (air), S. subtropicum (soil) and S. sympodiophorum (soil) were isolated from soil, marine water, rock, decaying wood and air (Zare and Gams 2001; Liu and Cai 2012; Nonaka et al. 2013; Liang et al. 2017). Simplicillium filiforme and S. coffeanum were isolated as endophytic fungi from plants (Crous et al. 2018; Gomes et al. 2018). Simplicillium lamellicola belongs to the hyperparasite fungi (Shin et al. 2017). Simplicillium lanosoniveum was reported as both an endophytic and hyperparasite fungi (Baiswar et al. 2014). It has been reported that Simplicillium is pathogenic to insects. Unfortunately, there are limited reports of insect-related Simplicillium.

The hosts of Simplicillium cicadellidae and S. lepidopterorum were larvae of Cicadidae and Lepidoptera, which feed through piercing-sucking and chewing. Moreover, S. formicidae was isolated from an infected ant. These three strains are likely to receive nutrients from plants (including living plants and plant residues) and animals (especially insects) based on the evolutionary pattern of Hypocreales. Simplicillium cicadellidae, S. formicidae and S. lepidopterorum represent three new species based on their nutritional preferences. To our knowledge, this is the first report of insect-associated Simplicillium species.

ITS and LSU have been widely used in the identification of Simplicillium (Liu and Cai 2012; Nonaka et al. 2013; Zhang et al. 2017; Sliva et al. 2018). In the present study, the combined dataset (ITS+LSU) was used to analysis of phylogenetic relationships among the new taxa and other Simplicillium species. Additionally, RPB1, RPB2 and TEF loci were added to analysis that the relationship among Simplicillium and its allies. The new species clustered with other Simplicillium species in a clade (Fig. 1), and this was consistent with morphological characteristics based identification. Six strains were clustered into three subclades (Fig. 2) and were distinctly different from other reported Simplicillium spp. Additionally, three species, S. chinense, S. coffeanum and 
S. filiforme were clustered in a subclade, and these species were associated with plants. This may be because of their nutritional preferences. Therefore, S. cicadellidae, $S$. formicidae and S. lepidopterorum are based on morphological characteristics, ecological characteristics and a phylogenetic analysis.

\section{Acknowledgements}

This work was supported by the National Natural Science Foundation of China (Grant No. 31460010, 31860002), the Doctoral Fund of Guiyang University of Chinese Medicine (3043-043170023), the National first-class construction discipline in Guizhou province (Chinese medical science) (GNYL[2017]008), and Engineering Research Center of General Higher Education in Guizhou Province (Qianjiaohe(2015)337). We thank Dr. Lesley Benyon, from Liwen Bianji and Edanz Group China (http:// www.liwenbianji.cn/ac), for editing the English text of a draft of this manuscript.

\section{References}

Baiswar P, Ngachan SV, Rymbai H, Chandra S (2014) Simplicillium lanosoniveum, a hyperparasite on Aecidium elaeagni-latifoliae in India. Australasian Plant Disease Notes 9(1): 144. https://doi.org/10.1007/s13314-014-0144-z

Castlebury LA, Rossman AY, Sung GH, Hyten AS, Spatafora JW (2004) Multigene phylogeny reveals new lineage for Stachybotrys chartarum, the indoor air fungus. Mycological Research 108: 864-872. https://doi.org/10.1017/S0953756204000607

Chen RS, Huang CC, Li JC, Tsay JG (2008) First report of Simplicillium lanosoniveum causing brown spot on Salvinia auriculata and S. molesta in Taiwan. Plant Disease 92(11): 1589-1589. https://doi.org/10.1094/PDIS-92-11-1589C

Chen RS, Huang CC, Li JC, Tsay JG (2017) Evaluation of characteristics of Simplicillium lanosoniveum on pathogenicity to aphids and in vitro antifungal potency against plant pathogenic fungi. International Journal of Environmental \& Agriculture Research 3 (1): 2454-1850.

Crous PW, Luangsa-ard JJ, Wingfield MJ, Carnegie AJ, Hernández-Restrepo M, Lombard L, Roux J, Barreto RW, Baseia IG, Cano-Lira JF, Martín MP, Morezova OV, Stchigel AM, Summerell BA, Brandrud TE, Dima B, Garcia D, Giraldo A, Guarro J, Gusmão LFP, Khamsuntorn P, Noordeloos ME, Nuankaew S, Pinruan U, Rodríguez-Andrade E, SouzaMotta CM, Thangavel R, van lperen AL, Abreu VP, Accioly T, Alves JL, Andrade JP, Bahram M, Baral HO, Barbier E, Barnes CW, Bendiksen E, Bernard E, Bezerra JDP, Bezerra JL, Bizio E, Blair JE, Bulyonkova TM, Cabral TS, Caiafa MV, Cantillo T, Colmán AA, Conceição LB, Cruz S, Cunha AOB, Darveaux BA, da Silva AL, da Sliva GA, da Sliva GM, da Sliva RMF, de Oliveira RJV, Oliveira RL, De Souza JT, Dueñas M, Evans HC, Epifani F, Felipe MTC, Fernández-López J, Ferreira BW, Fugueiredo CN, Filippova NV, Flores JA, Gené J, Ghorbani G, Gibertoni TB, Glushakova AM, Healy R, Huhndorf SM, IturrietaGonzález I, Javan-Nikkhah M, Juciano RF, Jurjević Ž, Kachalkin AV, Keochanpheng K, 
Krisai-Greilhuber I, Li YC, Lima AA, Machado AR, Madrid H, Magalhães OMC, Marbach PAS, Melanda GCS, Miller AN, Mongkolsamrit S, Nascimento RP, Oliveira TGL, Ordoñez ME, Orzes R, Palma MA, Pearce CJ, Pereira OL, Perrone G, Peterson SW, Pham THG, Piontelli E, Pordel A, Quijada L, Raja HA, Rosas de Paz E, Ryvarden L, Saitta A, Salcede SS, Sandoval-Denis M, Santos TAB, Seifert KA, Silva BDB, Smith ME, Soares AM, Sommai S, Sousa JO, Suetrong S, Susca A, Tedersoo L, Telleria MT, Thanakitpipattana D, Valenzuela-Lopez N, Visagie CM, Zapata M, Groenewald JZ (2018) Fungal Planet description sheets: 785-867. Persoonia: Molecular Phylogeny and Evolution of Fungi 41: 1-238. https://doi.org/10.3767/persoonia.2018.41.12

Curran J, Driver F, Ballard JWO, Milner RJ (1994) Phylogeny of Metarhizium: analysis of ribosomal DNA sequence data. Mycological Research 98: 547-552. https://doi.org/10.1016/ S0953-7562(09)80478-4

Dai Y, Lin Y, Pang X, Luo X, Salendra L, Wang JF, Zhou XF, Lu YJ, Yang B, Liu Y (2018) Peptides from the soft coral-associated fungus Simplicillium sp. SCSIO41209. Phytochemistry 154: 56-62. https://doi.org/10.1016/j.phytochem.2018.06.014

Dong Q, Dong R, Xing X, Li Y (2018) A new antibiotic produced by the cyanobacterium-symbiotic fungus Simplicillium lanosoniveum. Natural Product Research 32(11): 1348-1352. https://doi.org/10.1080/14786419.2017.1343320

Drummond A, Rambaut A (2007) BEAST: Bayesian evolutionary analysis by sampling trees. BMC Evolutionary Biology 7: 1-214. https://doi.org/10.1186/1471-2148-7-214

Fukuda T, Sudoh Y, Tsuchiya Y, Okuda T, Igarashi Y (2014) Isolation and biosynthesis of preus$\sin$ B, a pyrrolidine alkaloid from Simplicillium lanosoniveum. Journal of Natural Products 77 (4): 813-817. https://doi.org/10.1021/np400910r

Gauthier NW, Maruthachalam K, Subbarao KV, Brown M, Xiao Y, Robertson CL, Schneider RW (2014) Mycoparasitism of Phakopsora pachyrhizi, the soybean rust pathogen, by Simplicillium lanosoniveum. Biological Control 76: 87-94. https://doi.org/10.1016/j.biocontrol.2014.05.008

Gomes AA, Pinho DB, Cardeal ZL, Menezes HC, De Queiroz MV, Pereira OL (2018) Simplicillium coffeanum, a new endophytic species from Brazilian coffee plants, emitting antimicrobial volatiles. Phytotaxa 333(2): 188-198. https://doi.org/10.11646/phytotaxa.333.2.2

Huang Z, Yan SZ, Chen SL (2015) Optimization on fermentation conditions of Simplicillium obclavatum YX016 for the production of anthraquinones. Food Science and Technology 7: 3 pp.

Katoh K, Standley DM (2013) MAFFT multiple sequence alignment software version 7: improvements in performance and usability. Molecular Biology and Evolution 30(4): 772780. https://doi.org/10.1093/molbev/mst010

Liang JD, Han YF, Zhang JW, Du W, Liang ZQ, Li ZZ (2009) Optimal culture conditions for keratinase production by a novel thermophilic Myceliophthora thermophila strain GZUIFRH49-1. Journal of Applied Microbiology 110: 871-880. https://doi.org/10.1111/j.13652672.2011.04949.x

Liang X, Nong XH, Huang ZH, Qi SH (2017) Antifungal and antiviral cyclic peptides from the deep-sea-derived fungus Simplicillium obclavatum EIODSF 020. Journal of Agricultural and Food Chemistry 65 (25): 5114-5121. https://doi.org/10.1021/acs.jafc.7b01238 
Liang X, Zhang XY, Nong XH, Wang J, Huang ZH, Qi SH (2016) Eight linear peptides from the deep-sea-derived fungus Simplicillium obclavatum EIODSF 020. Tetrahedron 72(22): 3092-3097. https://doi.org/10.1016/j.tet.2016.04.032

Lim SY, Lee S, Kong HG, Lee J (2014) Entomopathogenicity of Simplicillium lanosoniveum isolated in Korea. Mycobiology 42(4): 317-321. https://doi.org/10.5941/ MYCO.2014.42.4.317

Liu F, Cai L (2012) Morphological and molecular characterization of a novel species of Simplicillium from China. Cryptogamie, Mycologie 33(2): 137-145. https://doi.org/10.7872/ crym.v33.iss2.2012.137

Mongkolsamrit S, Noisripoom W, Thanakitpipattana D, Wutikhun T, Spatafora JW, Luangsaard J (2018) Disentangling cryptic species with isaria-like morphs in Cordycipitaceae. Mycologia 110(1): 230-257.

Nonaka K, Kaifuchi S, Ōmura S, Masuma R (2013) Five new Simplicillium species (Cordycipitaceae) from soils in Tokyo, Japan. Mycoscience 54(1): 42-53. https://doi.org/10.1016/j. myc.2012.07.002

Qu JJ, Yu LQ, Zhang J, Han YF, Zou X (2018) A new entomopathogenic fungus, Ophiocordyceps ponerus sp. nov., from China. Phytotaxa 343(2): 116-126. https://doi.org/10.11646/phytotaxa.343.2.2

Ronquist F, Teslenko M, van der Mark P, Ayres DL, Darling A, Höhna S, Larget B, Liu L, Suchard MA, Huelsenbeck JP (2012) MrBayes 3.2: efficient Bayesian phylogenetic inference and model choice across a large model space. Systematic Biology 61: 539-542. https://doi.org/10.1093/sysbio/sys029

Roy S, Dutta T, Sarkar TS, Ghosh S (2013) Novel xylanases from Simplicillium obclavatum MTCC 9604: comparative analysis of production, purification and characterization of enzyme from submerged and solid state fermentation. SpringerPlus 2(1): 382. https://doi. org/10.1186/2193-1801-2-382

Shin TS, Yu NH, Lee J, Choi GJ, Kim JC, Shin CS (2017) Development of a biofungicide using a mycoparasitic fungus Simplicillium lamellicola BCP and its control efficacy against gray mold diseases of tomato and ginseng. The Plant Pathology Journal 33(3): 337. https://doi.org/10.5423/PPJ.FT.04.2017.0087

Skaptsov M, Smirnov S, Kutsev M, Uvarova O, Sinitsyna T, Shmakov A, Matsyura A (2017) Pathogenicity of Simplicillium lanosoniveum to Coccus hesperidum. Ukrainian Journal of Ecology 7 (4): 689-691. https://doi.org/10.15421/2017_1801

Spatafora JW, Sung GH, Sung JM, Hywel-Jones NL, White JF (2007) Phylogenetic evidence for an animal pathogen origin of ergot and the grass endophytes. Molecular Ecology 16: 1701-1711. https://doi.org/10.1111/j.1365-294X.2007.03225.x

Stamatakis A (2014) RAxML version 8: a tool for phylogenetic analysis and post-analysis of large phylogenies. Bioinformatics 30: 1312-1313. https://doi.org/10.1093/bioinformatics/btu033

Sung GH, Hywel-Jones NL, Sung JM, Luangsa-ard JJ, Shrestha B, Spatafora JW (2007) Phylogenetic classification of Cordyceps and the clavicipitaceous fungi. Studies in Mycology 57: 1-64. https://doi.org/10.3114/sim.2007.57.01 
Swofford DL (2002) PAUP* 4.0b10: phylogenetic analysis using parsimony (*and other methods). Sinauer, Sunderland.

Takata K, Iwatsuki M, Yamamoto T, Shirahata T, Nonaka K, Masuma R, Hayakawa Y, Hanaki H, Kobayashi Y, Petersson GA, Ōmura S, Shiomi K (2013) Aogacillins A and B produced by Simplicillium sp. FKI-5985: new circumventors of arbekacin resistance in MRSA. Organic Letters 15(18): 4678-4681. https://doi.org/10.1021/ol401975z

Tamura K, Stecher G, Peterson D, Filipski A, Kumar S (2013) MEGA6: molecular evolutionary genetics analysis version 6.0. Molecular Biology and Evolution 30: 2725-2729. https:// doi.org/10.1093/molbev/mst197

Uchida R, Kondo A, Yagi A, Nonaka K, Masuma R, Kobayashi K, Tomoda H (2019) Simpotentin, a new potentiator of amphotericin B activity against Candida albicans, produced by Simplicillium minatense FKI-4981. The Journal of Antibiotics 72(3): 134 pp. https://doi. org/10.1038/s41429-018-0128-x

Vaidya G, Lohman DJ, Meier R (2011) SequenceMatrix: concatenation software for the fast assembly of multi-gene datasets with character set and codon information. Cladistics 27(2): 171-180. https://doi.org/10.1111/j.1096-0031.2010.00329.x

van den Brink J, Samson RA, Hagen F, Boekhout T, de Vries RP (2012) Phylogeny of the industrial relevant, thermophilic genera Myceliophthora and Corynascus. Fungal Diversity 52: 197-207. https://doi.org/10.1007/s13225-011-0107-z

Ward NA, Robertson CL, Chanda AK, Schneider RW (2012) Effects of Simplicillium lanosoniveum on Phakopsora pachyrhizi, the soybean rust pathogen, and its use as a biological control agent. Phytopathology 102(8): 749-760. https://doi.org/10.1094/PHYTO-01-11-0031

White TJ, Bruns T, Lee S, Taylor J (1990) Amplification and direct sequencing of fungal ribosomal RNA genes for phylogenetics. In: Innis MA, Gelfand DH, Sninsky JJ, White TJ (Eds) PCR protocols: a guide to methods and applications. Academic Press, New York. 315-322. https://doi.org/10.1016/B978-0-12-372180-8.50042-1

Yan B, Fang ST, Li WZ, Liu SJ, Wang JH, Xia CH (2015) A new minor diketopiperazine from the sponge-derived fungus Simplicillium sp. YZ-11. Natural Product Research 29 (21): 2013-2017. https://doi.org/10.1080/14786419.2015.1027890

Zare R, Gams W (2001) A revision of Verticillium section Prostrata. IV. The genera Lecanicillium and Simplicillium gen. nov. Nova Hedwigia 73: 1-50.

Zare R, Gams W (2008) A revision of the Verticillium fungicola species complex and its affinity with the genus Lecanicillium. Mycological Research 112 (7): 811-824. https://doi. org/10.1016/j.mycres.2008.01.019

Zhang ZF, Liu F, Zhou X, Liu XZ, Liu SJ, Cai L (2017) Culturable mycobiota from Karst caves in China, with descriptions of 20 new species. Persoonia: Molecular Phylogeny and Evolution of Fungi 39: 1 pp. https://doi.org/10.3767/persoonia.2017.39.01

Zhao D, Liu B, Li LY, Zhu XF, Wang YY, Wang JQ, Duan YX, Chen LJ (2013) Simplicillium chinense: a biological control agent against plant parasitic nematodes. Biocontrol Science and Technology 23 (8): 980-986. https://doi.org/10.1080/09583157.2013.809514

Zou X, Liu AY, Liang ZQ, Han YF, Yang M (2010) Hirsutella liboensis, a new entomopathogenic species affecting Cossidae (Lepidoptera) in China. Mycotaxon 111 (1): 39-44. https:// doi.org/10.5248/111.39 